\title{
Understanding Language MOOC Learners: The Issue of Capability Development
}

\author{
https://doi.org/10.3991/ijet.v14i11.10205 \\ Nikoletta Agonács ( $\left.{ }^{\varpi}\right)$, João Filipe Matos \\ Universidade de Lisboa, Lisboa, Portugal \\ nikoletta.agonacs@campus.ul.pt
}

\begin{abstract}
Having a clear understanding of the learner population has an impact on effective course design and delivery in any learning context. However, it has greater importance in a MOOC learning environment because of the high diversity of participants. Most of the review studies on MOOC research mention learner population as a recurrent theme. These studies focus on behaviours, performance, learner participation and interaction patterns, learner perceptions and preferences, learner experiences, motivation, demographics, and classifications of learners into subpopulations. Nevertheless, there is little knowledge about the learners' perceptions of their own learning and the learning process, which is a key element of learning according to heutagogy, the theory of self-determined learning. In our study, which is based on two theoretical pillars (heutagogy and the Common European Framework of Reference for Languages: Learning, Teaching, Assessment) we aimed at capturing traces of capability development through language MOOC learners' reflections and identifying learning activities that are perceived by learners as preferred activities for skill acquisition in a language MOOC learning environment. We designed two open-ended questions in a survey format that were administered in an Italian language MOOC offered by Wellesley College. We were able to capture traces of capability development through learners' reflections, and we also found that a) based on learners' perceptions the most preferred way of acquiring new skills is through receptive activity types; b) these activity types coincide with learners' perceptions of their own understanding (listening and reading) skill development.
\end{abstract}

Keywords-Capability development, heutagogy, language MOOC, learner population

\section{The Importance of Understanding The Learner Population in MOOCs}

Understanding better the learner population has great importance for effective course design and delivery in any teaching and learning context. However, in a Massive Open Online Course (MOOC) learning environment it becomes an even more pertinent issue [1]-[3]. As the MOOC learning environment can host hundreds of thousands of participants from different cultures, of different ages, and with a variety 
of social and educational backgrounds, it becomes not only highly interesting but also extremely important to better understand how those populations behave, act and think, both as learners and individuals in that particular learning environment [4].

In today existing review studies within the MOOC research area, learner population is identified as one of the most researched subtopics. One of the first MOOC research reviews [5] already found learner experience as one of the key categories to be considered and therefore established a category of "participant focused" which included ten research articles. Kennedy [6] also identified that one of the key themes in early research on MOOCs is learner engagement, motivation, and presence to reduce learner isolation. In later research, Veletsianos and Shepherdson [7] found that most of the studies included in their review $(83.6 \%)$ have a focus on topics related to learners or learning. The topics comprise learner behaviours, performance, learner participation and interaction, learner perceptions and preferences, learner experiences, motivation, demographics, and classifications of learners into subpopulations. Gašević et al. [8] also found that learners' participation, engagement, and behavioural patterns in MOOC research is a recurrent theme. Ebben and Murphy [9] established the subcategory of learning analytics in case of xMOOCs, where they classified studies into two groups: (a) the correlations between learner characteristics and achievement, and (b) the search for patterns of behaviour about how learners interact with course materials (e.g., clickstream data). Even a recently published review study [10], which comprehends research published between 2008 and 2015, reports on studies that focus on learner need, experiences, motivation and online interaction patterns.

After having a more in-depth look into the studies reported in those reviews and others that concern learner populations in MOOCs (for example [11], [12]), we understood that there is little knowledge about the learners' perceptions of their own learning and the learning process. According to heutagogy, the theory of self-determined learning [13], having knowledge about our own learning is a key element of learning. Gaining knowledge about their own learning permits learners to have a clear understanding of their learning needs and preferences. This knowledge, then, enables them to decide not only on how to learn but also on what to learn, which is a principle of heutagogy [13]. In heutagogy, learners become fully responsible for their own learning and have an active role in the whole learning process.

Heutagogy has been proposed as a potential theory for distance education, however, more research is needed to confirm the theory's viability in distance education [14]. The concept of MOOC fits in well with the heutagogical principles: the selfselected MOOC learner, open access to content, share and recycle knowledge, and non-linear learning path characterise both MOOCs and heutagogy [15]. Therefore, heutagogy is more than worthy to be considered when it comes to the MOOC learning environment. Understanding better the MOOC learners' perceptions of their own learning and development would have a significant contribution to the MOOC research field bringing in a new perspective for the analysis of MOOC learner populations.

In this study, we focused on collecting data from Language MOOC (LMOOC) participants about their perceptions of their own learning and development, more specifically of their capability development. Before presenting the aim and objectives of this 
study, we explain what capability means within heutagogy, and how we interpret capability in this study context.

\section{Theoretical Frameworks}

\subsection{Heutagogy and capability}

As mentioned before, in heutagogy, critical reflection is a core element of learning. Through critical reflection, learners gain knowledge both about what they have learnt and the way in which it has been learnt [16]. This metacognitive process gives learners a better understanding of their skills and competences, which is critical to capability development.

The concept of capability derives from Stephenson [17] and emerged in 1980 in the United Kingdom because of the need for capacity improvement of British organisations to keep up in a decreasing marketplace [13]. With globalisation, the workplace environment changed, and it became necessary for people to be more flexible and quick learners to be able to compete in an accelerated and continuously changing environment. Capability enables people to deal effectively with this chaotic environment. Capability designates a holistic attribute; while competence consists of knowledge and skills, capability comprises knowledge about how to learn, creativity, a high degree of self-efficacy, collaboration, and the capacity to apply competences in familiar as well as in novel situations [13]. Hase and Kenyon [13] argue that for understanding how to develop capable people and enable capability, heutagogy is required. Because of capability being such a holistic concept and because it requires full competency in skills [18], observing capability is not easy. The context of such study should be as broad as possible, and it would be necessary to observe participants in different contexts (in learning, work and private situations) and during a long enough period in order to be able to observe capability.

\subsection{Capability development and foreign language learning}

If we consider the above-explained holistic concept of capability, the viability of a study conducted in a closed learning environment such as a MOOC becomes impossible. Therefore, in this study, we use the concept of capability following Blaschke's adoption:

Competency can be understood as proven ability in acquiring knowledge and skills, while capability is characterized by learner confidence in his or her competency and, as a result, the ability "to take appropriate and effective action to formulate and solve problems in both familiar and unfamiliar and changing settings" [19, p. 1]. [...] When learners are competent, they demonstrate the acquisition of knowledge and skills; skills can be repeated, and knowledge retrieved. When learners are capable, skills and knowledge can be reproduced in unfamiliar situations. Capability is then the extension of one's own competence, and without competency there cannot be capability [14, p. 59-60]. 
In that sense, in this study, we refer to capability as one's ability to use the acquired skills and competences both in familiar and unfamiliar situations. Applying this idea to foreign language acquisition and use, making learners reflect on their language acquisition process, and language use helps them become more aware not only of their needs and preferences but also of their acquired competences. This knowledge about their own learning and competences and their confidence will enable them to give an adequate answer in unknown situations, and so they will become more capable foreign language users. Therefore, in this study, when we talk about foreign language use, we understand any online or face to face (formal) language course environment (such as a LMOOC) as a known situation, and any other environment external to a course or formal learning environment, as an unknown situation. Hence, we define capable foreign language users as those who acquire knowledge and skills (competence) inside a course environment but are also able to reproduce the acquired skills and knowledge outside of the known course context, in authentic environment, better to say in everyday (professional or private) life.

\subsection{Language use and language competences}

According to the Common European Framework of Reference for Languages: Learning, Teaching, Assessment (CEFR), language use and acquisition can be described as:

Language use, embracing language learning, comprises the actions performed by persons who as individuals and as social agents develop a range of competences, both general and in particular communicative language competences. They draw on the competences at their disposal in various contexts under various conditions and under various constraints to engage in language activities involving language processes to produce and/or receive texts in relation to themes in specific domains, activating those strategies which seem most appropriate for carrying out the tasks to be accomplished. The monitoring of these actions by the participants leads to the reinforcement or modification of their competences. [...] Competences are the sum of knowledge, skills and characteristics that allow a person to perform actions [20, p. 9].

The framework lists several general and communicative language competences to be developed during foreign language acquisition (see Table 1), however in this study we focus on only three of them: lexical and grammatical competences and sociocultural knowledge (see Table 1). The criterion for choosing these specific competences was the ease in perceiving and understanding them from the part of the learners, as we discovered in their reflections during analysis. The framework also provides a selfassessment orientation tool, which is aimed at helping learners profile their language skills. This tool presents easily interpretable categories: understanding (listening and reading), speaking (production and interaction), and writing. 
Table 1. General and communicative language competences according to the CEFR

\begin{tabular}{|c|c|}
\hline & General competences \\
\hline \multirow{3}{*}{ Declarative knowledge } & Knowledge of the world \\
\hline & Sociocultural knowledge \\
\hline & Intercultural awareness \\
\hline \multirow{2}{*}{ Skills and know-how } & Practical skills and know-how \\
\hline & Intercultural skills and know-how \\
\hline Existential competence & - \\
\hline \multirow{4}{*}{ Ability to learn } & Language and communication awareness \\
\hline & General phonetic awareness and skills \\
\hline & Study skills \\
\hline & Heuristic skills \\
\hline \multicolumn{2}{|c|}{ Communicative language competences } \\
\hline \multirow{5}{*}{ Linguistic competences } & Lexical competence \\
\hline & Grammatical competence \\
\hline & Semantic competence \\
\hline & Phonological competence \\
\hline & Orthographic competence \\
\hline \multirow{5}{*}{ Sociolinguistic competences } & Linguistic markers of social relation \\
\hline & Politeness conventions \\
\hline & Expressions of folk wisdom \\
\hline & Register differences \\
\hline & Dialect and accent \\
\hline Pragmatic competence & Functional competence \\
\hline
\end{tabular}

Moreover, the framework distinguishes the following communicative language activities:

- Productive activities (oral production — speaking and written production-writing)

- Receptive activities (aural reception-listening, visual reception-reading, and audio-visual reception-video)

- Interactive activities (spoken and written interaction)

In our study, we used the three competences mentioned above (lexical and grammatical competence and sociocultural knowledge) to interpret the data, and the categories from the self-assessment orientation tool and the categories of language activities as categories of analysis.

\section{Study Context and Methodology}

We defined two specific objectives in this study: a) to capture traces of capability development through learners' reflections and b) to identify which types of activities and tasks are perceived by the learners as the best for their own development. We constructed two open-ended questions that we implemented in a questionnaire format in the AP Italian Language and Culture MOOC offered by Wellesley College (Wellesley, Massachusetts, USA) in the edX platform. The College administered the 
questionnaire and provided us with disidentified data for analysis. The two openended questions were the following:

- Question 1: Have you used the newly acquired listening, speaking, reading, or writing skills out of the course environment? Write about this experience! (Where, when, and how you used them.)

- Question 2: Write about your learning experience of Module 1/2/3/4/5/6! (e.g., What did you like the most/the least?; Which activities were the best for you to acquire new skills?; How do you feel about the forum experience?; etc.)

The two questions were embedded in the course as optional after each of the six modules. Data collection process ran during October-November 2018. The reason for multiple times of data collection through the modules was to understand if there is an increase in capability development through the course. However, participation in the questionnaires was voluntary, and in the case of module 5 and 6 , no data was obtained. Therefore, the collected data did not permit us to make such comparison. For that reason, datasets collected from different modules were merged into one, and no comparison was carried out.

At the time of the closure of the questionnaire, the total number of enrolled learners on the AP Italian Language and Culture course was 7390; the number of active learners (those who at least once in that week performed an activity in the course) was 740; the number of learners who earned a passing grade was 31; and the number of those who finished all the activities was 11. Through the questionnaires, we had a total number of 90 responses: 46 for the first question and 44 for the second one. The total number of respondents of the two survey questions was 40 . We obtained demographic data only from $28 \%$ of the respondents (from 11 respondents). Hereby, we present the demographic data regarding those 11 respondents. However, we recognize that the data is not enough to fully characterise the sample population. The age distribution of those 11 respondents was between 22 and 73; there were respondents from Columbia, Croatia, England, Mexico, Netherlands, USA and Russia. The majority of the 11 respondents hold a master's degree, but there were two respondents with a doctorate and one respondent with a bachelor's degree. Regarding the respondents' professional occupation, five of them were retired, four of them indicated as employed (full-time, part-time or self-employed), and two of them as students. All those respondents have already experienced a MOOC before this course. All respondents' motivation for enrolling on this course was to get familiarised with the Italian language and culture, and two respondents also specified that their aim was to improve their Italian language (and cultural) knowledge and skills.

Concerning the data analysis process, we predefined the categories of analysis based on the CEFR. We applied a descriptive content analysis approach [21] due to our aim of tracking traces of capability development and identifying learning activities that are perceived as contributing to new skill acquisition.

We analysed the two datasets separately from Question 1 and Question 2. For Question 1, we predefined the categories and subcategories based on the selfassessment tool of the CEFR, which are presented in Figure 1. For Question 2, we used the communicative language activities, described, in detail, above as categories 
of analysis, and the lexical, grammatical competences and sociocultural knowledge to help us interpret the data.

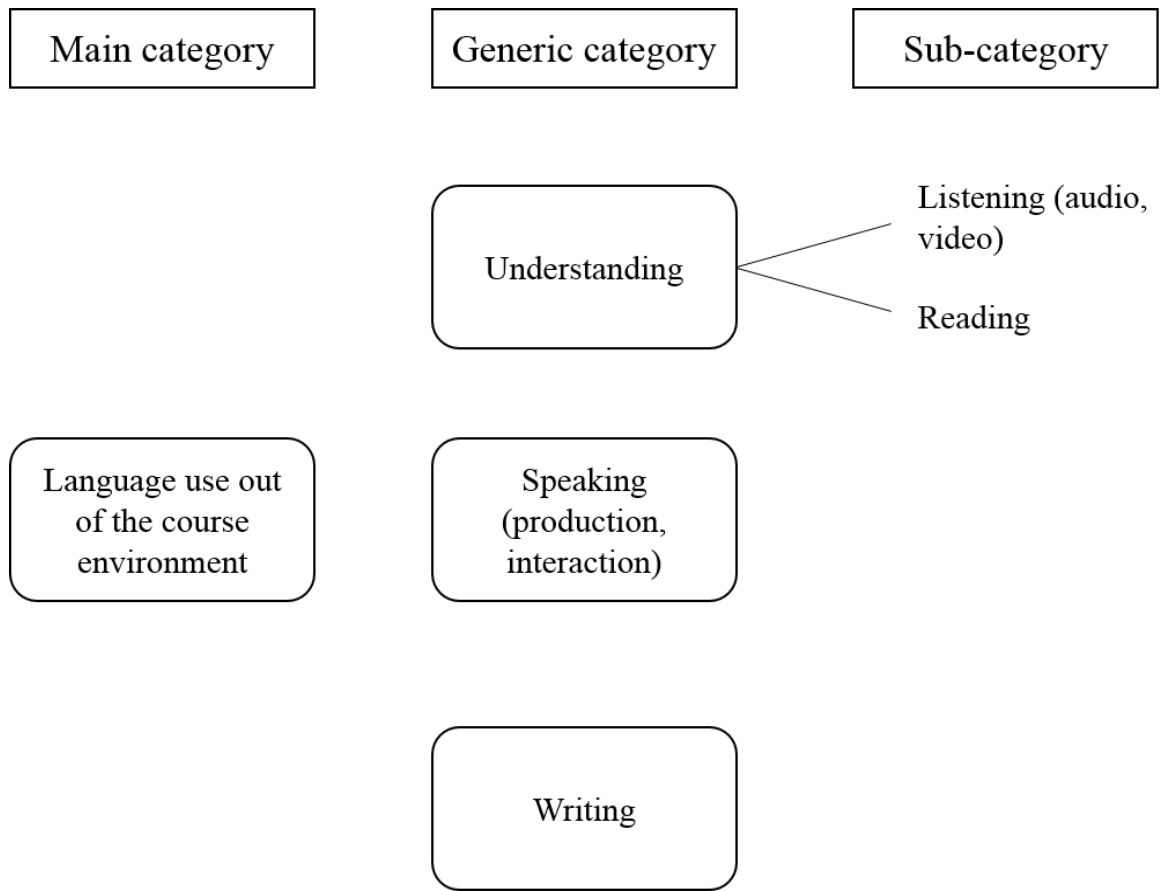

Fig. 1. Predefined categories for Question 1 based on the CEFR

\section{$4 \quad$ Results and Discussion ${ }^{1}$}

\subsection{Question 1. Capturing traces of capability development}

The first observation we made after having categorised the units of meaning is that there is a tendency to affirmative (yes) answer to the question if learners have used the newly acquired listening, speaking, reading or writing skills out of the course environment. Therefore, it seems to exist a perception of skill and knowledge (competence) acquisition from the part of the respondents. Though, we identified 15 responses out of 46 where an explicit negative answer (no) was given. In many of these answers, there is no or little specific justification of why respondents have not used the new skills. They gave answers such as No, I have not.; Unfortunately, I haven't. or No, I haven't yet. Just started the course. The negative answers were not categorised for analysis because of lacking references to specific skills and because of our objec-

\footnotetext{
${ }^{1}$ All the citations from participants are presented in their original format without any change in grammar, spelling, punctuation or vocabulary. Additional explanation was added to the original text where it was necessary.
} 
tive of tracing capability development through the reflections. In other cases, the reflection was general (Yes. I come to Italy frequently. or Yes, With my granddaughter attending an Italian school. or Yes. I live in Italy, so I always have to use these skills in everyday life!) and it was impossible to categorise these units of meaning through the predefined language skills. Therefore, these reflections were also excluded from the final analysis.

When we looked at the positive answers we choose for analysis, we also understood that there is a higher number of references in respondents' reflections for the two skills of understanding (listening and reading) than for speaking or writing. (We found the least reference for writing.) Regarding listening, respondents referred to podcasts, radio, lyrics or opera and regarding watching, they mentioned movies, series or Italian TV channels. In most of the answers, besides the evidence of the use of listening skills outside the course context, we noted recognition of self-progress by the respondents: I can watch Italian movies without subtitles now. It was impossible until three weeks ago!; or I have improved my listening and reading skills in Italian. I already can understand some lyrics of songs and videos. In the case of reading skills, we found some similar examples expressing the recognition of self-progress: I can now read Italian media; or I am now able to read any italian news/magazine/opinion/online material I like (except literature, that I haven't tried).

While in listening and reading all the units of meaning reveal an active use of skills and many times those are confirmed with evidence of use, in case of speaking and writing, answers are more heterogeneous. Besides the confirming answers (I have also been able to communicate adequately at a colloquial/everyday context during a recent trip to Northern Italy. or I often send emails to Italian recruiters.), we found answers that explicitly express not only lack of use (I don't speak or write in Italian.), but also recognition of lack of skills and need for improvement: Speaking is difficult and I need to practice more.; or I still need to improve the communication skills., and lack of motivation (I am not giving emphasis to speaking and writing.).

It is important to highlight that there are some recurrent themes that are worthy of a more in-depth analysis. One of these themes is opportunity. In some answers, respondents justified their negative answer with the lack of opportunity for language use (I did not have the opportunity to use them other than in the course.). Opportunity in language acquisition seems to have a strong relationship with capability development. If learners have opportunity for language use outside of the known course context means that they have opportunity to use the newly acquired skills in authentic environments; they have opportunity for capability development. With the actual use of language in an authentic environment, they will experience and learn about themselves that they are able to use the newly acquired skills. This recognition makes them conscious about their acquisition of skills and competences and will make them more confident for further use of those skills. Consequently, they will seek new opportunities where they can use those skills (capability development). Therefore, capability can emerge only if there is and if learners can find opportunity for language use outside the known (course) context. In case of some skills, though, it is easier to have or find opportunities for language use. Learners by watching Italian movies or reading Italian newspapers, magazines or books create an environment (external to a language 
course) for the development of understanding skills and can also easily perceive their self-progress as mentioned in the reflection hereby: I watch Italian shows and movies at home. I try to understand without the subtitles and sometimes find subtle differences that are not accurately reflected in the subtitles. While it is more challenging to create an authentic environment (external to a language course) for writing or speaking skills development due to the necessity of another person to communicate with, as mentioned here: I have had little opportunity to use any new skills I have required due, primarily. to a lack of opportunities to speak Italian with someone. Although, we found some units referring to the use of speaking and writing skills in authentic environments: I often send emails to Italian recruiters. or I have also participated on some Italian job interviews. or I have an Italian ragazzo [boyfriend] and I always let him check my answers. We talk [about] Italian food (he is from Toscana), Italian places.

The other recurrent theme is intention. In some reflections, respondents expressed a clear intention to use the acquired skills in future situations: I will be using the listening and speaking skills acquired when I go on holiday to visit relatives in Italy. When learners have a clear intention for future language use, it comes from a certain confidence in their skills and competences. Therefore, capability development might have happened, (even if in these cases we don't have clear evidence of capability as in the previous cases) considering the definition of capability we used in this study: "capability is characterized by learner confidence in his or her competency" [13, p. 59], and as a result, the ability to use their competences not only in familiar but also in unfamiliar situations.

Another theme to be mentioned is the recognition and consciousness of selfprogress. As mentioned before, in general respondents expressed a certain level of consciousness of their self-progress in their reflections, but it seems that it is not easy for everybody. We found an example of a direct expression of lack of understanding of self-progress: It is difficult to say. In addition to this course, I have a tutor (Skype), a conversation partner (Italian), and other online resources I have found, for example, "Learn with Lucrezia". My opportunity to speak Italian is limited. Where I acquire a skill is unclear to me.

Summarising, we were able to capture traces of capability development in learners' self-reflections since they presented evidence of language use in authentic situations and outside of the learning context (outside of the Language MOOC).

\subsection{Question 2. Identifying activities that contribute to competence development}

Our first observation regarding the second question was that there was a tendency to identify receptive activities (both listening and reading) as preferred activities. For example, answers as I like very much the video activities.; I really like the listening part; The videos (students in Bologna, chefs, etc.) were all great. I also liked the videos with the inserted questions afterwards.; I liked reading / listening and then trying to answer the questions.; I love the interviews, the readings and listening to them.;.; etc. Some participants went beyond referring to these activities as preferred, they felt them helpful for new skill acquisition: I enjoyed listening and reading tasks as they 
helped me to improve my understanding of written and spoken Italian; [...] both the listening and reading exercises were helpful to continue to improve my language skills.; Listening first then eventually reading the script is most helpful for me as I find understanding spoken Italian my biggest challenge.; The listening activities were the most difficult ones, but also the ones that helped me the most to acquire new skills.; The interviews and audio exercises helped me the most as they exposed me to native speakers.; I liked the readings, the videos, everything brought me closer to know and handle more this language. The combination of audio-visual and visual activities was perceived as very useful: Listening to video while reading the accompanying text I believe is very helpful. or Mostly I like the combination of audio and pdf files, I find more effective way to learn.; [I appreciated and enjoyed] The videos with text.

Productive activities (both speaking and writing) were scarcely referred, however when it was referred it was perceived positive: the "scriviamo" [writing] exercise is very useful. Regarding the interactive (speaking) activity, references were almost absent, the only feedback concerned the quality of the activity: The "conversiamo" [speaking] section is not particularly practical (too cumbersome).

Participants did not engage in the interactive (writing) activity (forum activity). Most answers expressed the lack of participation due to a variety of reasons: I do not participate due to the lack of time.; I did not engage the forum discussions.; I don't participate in forum discussions, don't really like social media style.; I participate little in the forum. Some of the participants did not find the forum activity useful: I do not find the forum particularly helpful.; The forum experience does not give me much.; I am not really using the forums, I am sorry: but they might be helpful to many people to learn the language while socializing too. Others expressed intention to participate in the future suggesting that they recognise some sort of role in that activity: I haven't, at this point, participated in the forum, mainly because I am short of time and when I have taken other courses I found that forum activity does not repay the time that it takes. But I might try later on.; Sorry to say I'm not sure what the 'forum" experience is. I'll look more closely for this feature in the next sessions. Nevertheless, we found positive reflections about the forum experience of participants who engaged and suggested further and deeper development of the discussions: The forum is amazing, all the resources and comments are very helpful!; The forum experience is very good because it makes you write, which for me is the most difficult part of learning Italian.; Other than that I like the discussion forum because it makes me use the language - at least in writing -, but unfortunately the discussions there do not really take on a life of their own.

It should be highlighted, that in the course, all (receptive) reading, and listening exercises were related to lexical competences. The exercises related mostly to vocabulary and written or spoken comprehension. The grammar exercises were placed at the end of each module and were optional. In the reflections, besides the references to learning activities, we found positive references to some of the linguistic (grammatical or lexical) competences: It was a good mixture for me of reviewing verbs and grammar and learning some new idiom.; I love the interactive learning with the LEARN vocabulary. The quizzes and the [grammar] review exercises are also very helpful.; I like the grammar module, but I prefer to start with it. In most of the units of 
meaning, we identified references either to learning activities or to competences. However, in one case we could also identify a direct connection between the learning activity and the acquired language competence, which is related to receptive activity and lexical competence: I mostly liked working on texts, because I learnt some vocabulary. In another reflection, a connection between activity and competence can be captured though, there is a contradiction in the learner's objective of developing speaking skills and the chosen learning path of receptive learning activities: I liked to reading / listen and then trying to answer the questions. I did not try the speaking or writing exercises. I am an adult (senior), trying to be able to speak in Italian. My reading comprehension has improved but my speaking ability remains stagnant.

It seems that there is a tendency in learners' preference for receptive (visual, aural, or audio-visual) activities. When we discuss these results, it is important to emphasise that while the receptive activities were all graded, the productive and interactive (both oral and written) and the grammar activities were optional. This fact can have a significant impact both on participants' perceptions and motivations, which cannot be ignored. However, this condition would have a higher impact in cases where learners' objective is to earn a certificate (which they pay for). For learners who have no such objective, participation in any activity is optional. In this study, we did not collect data on earning certificates; therefore, we do not have enough information to compare the results of learners motivated to earn a certificate with the results of learners without this motivation. For that reason, our conclusion about learners' preference for receptive activities is not final. What we understood in this study is that a) speaking or writing activities were barely commented, or the comments explicitly expressed lack of participation, and b) receptive activities appeared to be preferred also in cases when the learner's objective was not understanding language but producing or eventually interacting. Moreover, the references to receptive activities were homogeneously positive.

The receptive activities focused mostly on lexical competences, however for participants grammatical competences seem to be similarly important (The grammar review was very helpful; The grammar revision is good. It would be nice though not to put grammar issues only at the end of the module. Why not sprinkle a bit of grammar in the middle of the module?; I like the grammar module, but I prefer to start with it.).

In addition to the linguistic competences, we understood that many reflections contained elements referring to sociocultural knowledge: [I enjoyed] to learn more about Italian culture; The overview of Italian geography was also very interesting and useful. I had never thought about the whole country of Italy in that structured way before.; My favorite lessons are those that touch on features of Italian life and history: the Renaissance, art, culture, general statistics, and food. My affinity for all things Italian has been nicely enhanced thanks to this wide-ranging class.; I think tying in Italian culture is a great way to learn while making the lessons interesting.; All the themes were interesting. I feel I know a bit more about Italy and its culture; etc. These answers demonstrate that a) there was a gain of sociocultural knowledge about Italy and Italian culture, and b) linking sociocultural knowledge to language competences in a language course has a motivating force to language learning. 
In conclusion, we were able to identify preferred learning activities in the learners' reflections. However, in most cases, reflections were not deep enough to permit us to recognise and make conclusions about activities which learners think contributed most to their skill development. We saw that there is a tendency in learners' preferences towards receptive activities, though the reason for that is not clear since there are several conditions that might influence the learners' preferences. Further research is needed to identify the reasons behind learners' preferences in order to confirm the tendency. In addition, little evidence was found in the reflections where a connection between activity type and competence was (or could be) established.

\section{Conclusion and Future Work}

The hereby presented analysis and results are not entirely conclusive. Our objective was to outline some essential points about learners' perceptions of their own learning and capture traces of capability development in an Italian Language MOOC.

We found that based on learners' perceptions the most preferred way of acquiring new skills is through receptive (aural, visual or audio-visual) activity types or their combination. Reasons behind this preference should be further investigated and clarified. We also understood that the selected types coincide with learners' perceptions of their understanding (listening and reading) skill development. We were also able to capture traces of capability development through learners' reflections since they provided examples of evidence of language use in unfamiliar and authentic situations (outside of the course context). Activities that learners most perform or search for outside the course environment are also receptive activity types. We pointed out that in language learning capability has a strong relationship with opportunity: without opportunities for language use in authentic environments capability cannot emerge.

This study has some limitations. We must acknowledge that our conclusions are based on learners' perceptions of their own learning, which might not coincide with their real level of acquired skills and competences. For this reason, further studies in this area should first measure the learners' level of self-reflection and selfconsciousness so that we can deeper understand their progress. Moreover, their learning activity inside the course should be observed, and data on their activity (results and engagement) should be collected in order to compare their perceptions with their actual engagement in the course. Analysing those kinds of data could give a more comprehensive understanding of capability development.

We must keep in mind that the receptive exercises were all linked to lexical activities and were all and graded, while the grammar and speaking or writing activities were optional. This fact might have an impact on our results since earning points might be a motivation for learners to engage in course activities. Future studies, therefore, should be carried out in Language MOOC environments where graded activities include a) receptive, productive and interactive activities and b) focus on both lexical and grammatical competencies.

Despite its limitations, this study has a significant contribution to the MOOC research field inasmuch as it focuses on the learners' perceptions of their own learning 
and development-a barely researched aspect of the learner population of MOOCsby bringing the idea of heutagogy and its principles into the discussion of MOOCs.

\section{Acknowledgement}

This article reports research developed within the PhD Program Technology Enhanced Learning and Societal Challenges, funded by Fundação para a Ciência e Tecnologia, FCT I. P. - Portugal, under contracts \# PD/00173/2014 and $\mathrm{PD} / \mathrm{BD} / 135196 / 2017$. We thank Wellesley College for having ensured us the possibility to carry out our research in the AP Italian Language and Culture MOOC. We would like to give a special thanks to Daniela Bartalesi-Graf, Lecturer in Italian studies, to David O'Steen, Director, Research \& Instructional Support and Associate Director, Blended Learning Initiative, and Wellesley's Office of Institutional Research and the Office of the Provost.

\section{$7 \quad$ References}

[1] C. Milligan, A. Littlejohn, and A. Margaryan, "Patterns of engagement in connectivist MOOCs," J. Online Learn. Teach., vol. 9, no. 2, pp. 149-159, 2013.

[2] J. Haywood, "No such thing as a free MOOC," 2012. [Online]. Available: https://www.jisc.ac.uk/blog/no-such-thing-as-a-free-mooc-20-jul-2012.

[3] S. Zheng, M. B. Rosson, P. C. Shih, and J. M. Carroll, "Understanding student motivation, behaviors, and perceptions in MOOCs," in Proc.18th ACM Conf. Computer Supported Cooperative Work \& Social Computing - CSCW '15, 2015, pp. 1882-1895. https://doi.org/10.1145/2675133.2675217

[4] J. S. Tahiri, S. Bennani, and M. K. Idrissi, "diffMOOC: Differentiated learning paths through the use of differentiated instruction within MOOC," Int. J. Emerg. Technol. Learn., vol. 12, no. 3, pp. 197-218, Mar. 2017. https://doi.org/10.3991/ijet.v12i03.6527

[5] T. R. Liyanagunawardena, A. A. Adams, and S. A. Williams, "MOOCs: A systematic study of the published literature 2008-2012," The Int. Rev. Res. Open Distributed Learn., vol. 14, no. 3, Jul. 2013 https://doi.org/10.19173/irrodl.v14i3.1455

[6] J. Kennedy, "Characteristics of Massive Open Online Courses (MOOCs): A Research Review, 2009-2012,” J. Interact. Online Learn., vol. 13, no. 1, pp. 1-16, 2014. Available: https://eric.ed.gov/?id=EJ1032981

[7] G. Veletsianos and P. Shepherdson, "A systematic analysis and synthesis of the empirical MOOC literature published in 2013-2015," The Int. Rev. Res. Open Distributed Learn., vol. 17, no. 2, pp. 198-221, Mar. 2016. https://doi.org/10.19173/irrodl.v17i2.2448

[8] D. Gašević, V. Kovanović, S. Joksimović, and G. Siemens, "Where is Research on Massive Open Online Courses Headed? A Data Analysis of the MOOC Research Initiative," The Int. Rev. Res. Open Distributed Learn., vol. 15, no. 5, pp. 134-176, Oct. 2014. doi: https://doi.org/10.19173/irrodl.v15i5.1954

[9] M. Ebben and J. S. Murphy, "Unpacking MOOC scholarly discourse: a review of nascent MOOC scholarship," Learn. Media Technol., vol. 39, no. 3, pp. 328-345, Jan. 2014. https://doi.org/10.1080/17439884.2013.878352 
[10] O. Zawacki-Richter, A. Bozkurt, U. Alturki, and A. Aldraiweesh, "What research says about MOOCs - An explorative content analysis," The Int. Rev. Res. Open Distributed Learn., vol. 19, no. 1, pp. 242-259, 2018. https://doi.org/10.19173/irrodl.v19i1.3356

[11] Y. Li, "Feature Extraction and Learning Effect Analysis for MOOCs Users Based on Data Mining," Int. J. Emerg. Technol. Learn., vol. 13, no. 10, pp. 108-121, Oct. 2018. doi: 10.12738/estp.2018.5.015. https://doi.org/10.3991/ijet.v13i10.9456

[12] J. Xi, Y. Chen, and G. Wang, "Design of a personalized massive open online course platform,” Int. J. Emerg. Technol. Learn., vol. 13, no. 4, pp. 58-70, Mar. 2018. https://doi.org/10.3991/ijet.v13i04.8470

[13] S. Hase and C. Kenyon, "From andragogy to heutagogy," UltiBase, pp. 1-7, 2000.

[14] L. M. Blaschke, "Heutagogy and lifelong learning: A review of heutagogical practice and self-determined learning," The Int. Rev. Res. Open Distance Learn., vol. 13, no. 1, pp. 5671, Jan. 2012. doi: https://doi.org/10.19173/irrodl.v13i1.1076

[15] N. Agonács and J. F. Matos, "Heutagogy and self-determined learning: A review of the published literature on the application and implementation of the theory," Open Learn. J. Open, Distance e-Learning, pp. 1-18, Jan. 2019. https://doi.org/10.1080/02680513.2018.1562329

[16] L. M. Blaschke and S. Hase, "Heutagogy: A Holistic Framework for Creating TwentyFirst-Century Self-determined Learners," in Lecuter Notes in Education Technology, pp. 25-40, Jul. 2016. https://doi.org/10.1007/978-3-662-47724-3 2

[17] J. Stephenson, "Capability and Competence: Are they the same and does it matter?," Capability, vol. 1, no. 1, pp. 3-4, 1994.

[18] L. M. Blaschke, "Using social media to engage and develop the online learner in selfdetermined learning.," Res. Learn. Technol., vol. 22, no. 1, pp. 1-23, Nov. 2014. https://doi.org/10.3402/rlt.v22.21635

[19] L. Cairns, “Capability: going beyond competence," Capability, vol. 2, p. 80, 1996.

[20] Council of Europe. "Common European Framework of Reference for Languages: Learning, Teaching, Assessment," Cambridge, U.K.: Press Syndicate of the University of Cambridge, 2001.

[21] K. A. Neuendorf, The Content Analysis Guidebook. SAGE Publications, 2002.

\section{Authors}

Nikoletta Agonács is a PhD candidate of the FCT Technology Enhanced Learning and Societal Challenges Doctorate Program at the Institute of Education of the University of Lisbon. She holds a bachelor's degree in Romance Philology, specialising in Italian Studies, and a master's degree in Foreign Language Teaching (Italian and Hungarian) from the University of Eötvös Loránd, Budapest. She is an invited researcher and instructional designer at the e-Learning Lab of the University of Lisbon. Previously, she worked as a Learning Technologies Specialist being involved in online course design and in elaboration of proposals for various ICT-related international public tenders in the field of education mainly for Africa, Asia, and Latin America.

João Filipe Matos is a Full Professor at the Institute of Education of the University of Lisbon (IEULisboa). He holds a bachelor's (1982) and a master's (1986) degree in Mathematics, a doctorate (1991) degree, and aggregation (2000) in Education. He is the Director of the Doctorate Program FCT Technology Enhanced Learning and Soci- 
etal Challenges, the Doctoral course in ICT in Education, the Master's Degree Program in e-Learning and Distance Education, and the Master's Degree Program in Computer Education. He gives lectures in research methodologies in distance education and e-Learning. He coordinates the Centre for Competence in Technology and Innovation (C2TI) of IEULisboa aimed at supporting initiatives of schools and teachers in the field of digital technologies in collaboration with the Ministry of Education. $\mathrm{He}$ is part of the e-Learning Lab coordination team within the framework of the eLearning Project of the rectorate of ULisboa.

Article submitted 2019-01-25. Resubmitted 2019-03-01. Final acceptance 2019-03-02. Final version published as submitted by the authors. 